\title{
DISCURSO Y EXPRESIONES DISCURSIVAS DEL PROFESORADO ¿CÓMO SE REPRODUCEN LOS ESTEREOTIPOS DE GÉNERO?
}

\author{
DISCOURSE AND DISCURSIVE EXPRESSIONS OF THE TEACHING \\ STAFF HOW ARE GENDER S'TEREOTYPES REPRODUCED? \\ (iDeorgette Vargas Huanca ${ }^{*}$, (D) Ítalo Jaime Quispe Pérez ${ }^{1}$ \\ gvargas@esan.edu.pe; Italo.quispe@uarm.edu.pe \\ ${ }^{1}$ Universidad Antonio Ruiz de Montoya, Lima, Perú
}

*Correspondencia: Georgette Vargas Huanca. Email: gvargas@esan.edu.pe

Recibido: 05.07.19 | Aprobado: 11.09.19

\section{RESUMEN}

Las investigaciones realizadas sobre cómo los estereotipos de género se reproducen en la relación profesorado-alumnado de la EBR tienen como objeto de estudio el discurso oral de profesores y profesoras, el cual no explica cómo se siguen reproduciendo los estereotipos de género en las escuelas a pesar del cuidado que el profesorado, consciente de este problema, le otorga a su relación jerárquica con el alumnado. El objetivo del presente artículo es argumentar que si consideramos la relación profesorado-alumnado como habitus, entonces la reproducción de los estereotipos de género en las escuelas se evidencia no solo en el discurso oral del profesorado, sino en las expresiones discursivas diversas.

Palabras clave: Discurso, Expresiones discursivas, Género, Violencia de género, Habitus.

\begin{abstract}
The research carried out on how gender stereotypes are reproduced in the EBR teacher-student relationship has as its object of study the oral discourse of male and female teachers, which does not explain how gender stereotypes continue to be reproduced in schools despite of the care that teachers, aware of this problem, give to their hierarchical relationship with students. The objective of this article is to argue that if we consider the teacher-student relationship as a habitus, then the reproduction of gender stereotypes in schools is evident not only in the oral discourse of teachers, but also in diverse discursive expressions.
\end{abstract}

Keywords: Discourse, Discursive expressions, Gender, Gender violence, Habitus. 


\section{INTRODUCCIÓN}

Las investigaciones realizadas sobre cómo los estereotipos de género se reproducen en la relación profesorado-alumnado de la EBR tienen como objeto de estudio el discurso oral de profesores y profesoras, el cual no explica cómo se siguen reproduciendo los estereotipos de género en las escuelas a pesar del cuidado que el profesorado, consciente de este problema, le otorga a su relación jerárquica con el alumnado. El objetivo del presente artículo es argumentar que si consideramos la relación profesorado-alumnado como habitus, entonces la reproducción de los estereotipos de género en las escuelas se evidencia no solo en el discurso oral del profesorado, sino en las expresiones discursivas diversas.

Las relaciones de género sostenidas en estereotipos se explican por las prácticas de discriminación, racismo y sexismo propios de una sociedad marcada por brechas históricas de desigualdad, como es la nuestra. Estas prácticas sociales surgen en un proceso de socialización que en que se atraviesa por diversas instituciones socializadoras como la familia o la escuela, entre otras. Desde la educación, se sostiene que evitar la socialización a través de las prácticas no deseables puede ayudar a evitarlas (Parker, 2010); sin embargo, las instituciones socializadoras están estructuradas y responden a una sociedad más amplia. El profesorado, por ejemplo, aunque cuide su discurso oral, no puede evitar reproducir las estructuras de la sociedad en que se desenvuelve por medio de expresiones discursivas como la ambientación de su aula, la ropa que utiliza, los materiales que elabora para el alumnado, las letras de canciones que elige para sus clases, etc. Estas expresiones del habitus debieran ser, sostenemos, los objetos de investigación para comprender cómo se reproducen y mantienen las estructuras sociales; por ejemplo, las que refieren a los estereotipos de género.

Es cierto que se ha investigado sobre la influencia de la escuela, como institución social, en el desarrollo cívico y moral de los sujetos, pero, aunque tales investigaciones han dado paso a acciones encaminadas a disminuir la violencia social desde el colegio, el problema persiste. La preocupación por esta situación nos conduce a cuestionar en qué medida, no solo el discurso, sino también las expresiones discursivas reproducen asimetrías y relaciones de discriminación. En lo que sigue, pretendemos explicar la base teórica de las investigaciones que toman como objeto de estudio las expresiones discursivas que van más allá de las expresiones orales del profesorado.

\section{HABITUS, VIOLENCIA SIMBÓLICA Y MERCADO LINGÜÍSTICO}

En términos de Bourdieu, el habitus se define como:

“(...) un sistema de disposiciones durables y transferibles -estructuras estructuradas predispuestas a funcionar como estructuras estructurantes- que integran todas las experiencias pasadas y funciona en cada momento como matriz estructurante de las percepciones, las apreciaciones y las acciones de los agentes cara a una coyuntura o acontecimiento y que él contribuye a producir (Bourdieu, 1990, p. 92).

No se trata de una posesión o de una postura, sino que puede ser entendido como la posibilidad de volverse sujeto social, y antes de ello no existe un pre-babitus. Todo sujeto, por ser social, implica eo ipso, un habitus. Los sujetos sociales, podría decirse, no poseen un 
habitus, son habitus ${ }^{1}$. En esta dinámica de interacción, el proceso de adhesión a la estructura social se naturaliza, se normaliza y se "acepta" el orden de las cosas en un "deber ser". Hay una aceptación dóxica de las estructuras objetivas y las cognitivas, debido a que los agentes sociales, a través de las expresiones discursivas "educan” la percepción del sujeto y su reconocimiento social, que puede ser a través del lenguaje, la educación y la clasificación social (Bourdieu, 2000). Entonces, el habitus se convierte en parte constituyente del sujeto y no un añadido social, como su propio cuerpo físico. En palabras de Bourdieu:

"(...) la incorporación de las jerarquías sociales por medio de los esquemas del babitus, inclinan a los agentes, incluso a los más desventajados, a percibir el mundo como evidente y a aceptarlo como natural, más que a rebelarse con él, a oponerle mundo posibles, diferentes y aun, antagonistas: el sentido de la posición como sentido de loa que uno puede, o no, "permitirse" implica una aceptación tácita de la propia posición, un sentido de los límites o, lo que viene a ser lo mismo, un sentido de las distancias que se deben marcar o mantener, respetar o hacer respetar" (Bourdieu, 1990, p. 289).

El orden y la disposición de los "objetos" en el mundo son creaciones de mundos posibles, pero sobre la base de la proyección de un mundo anterior, de una cultura interiorizada según un habitus. El primer referente estructural al que está sometido el sujeto es la división socialmente construida de los sexos. Esta condensa las relaciones sociales de dominio y explotación entre hombres y mujeres tanto en lo físico como en lo mental que obligan al sujeto a dividir todo lo que lo rodea en este binomio estructural y entiende al mundo, así como a sus prácticas, en función de la oposición entre lo masculino y lo femenino. Entonces, el sistema se confirma y legitima por medio de las prácticas mismas del sujeto (Bourdieu, 2000). Luego, son las instituciones sociales las que hacen la "transmisión" de estas estructuras y se realizan en un "estado práctico", sin anunciaciones o explicación, sin acceder a un nivel discursivo. Es decir, su "aprehender" involucra un incorporar e introducir al cuerpo la práctica, no resulta consciente, sino que son las experiencias acumuladas las que le refieren al sujeto una determinada posición en el espacio social. Es ello lo que lo habilita para conformar determinadas disposiciones e inhabilita otras. Entonces, el habitus, incorporado ahora en el sujeto, produce prácticas, individuales y colectivas, conforme a los principios engendrados por sus experiencias o historicidad en la comunidad y que están pre adaptadas a las exigencias del poder dominante (Bourdieu, 2000).

Por otro lado, la violencia simbólica es "esa coerción que se instituye por mediación de una adhesión que el dominado no puede evitar otorgar al dominante (y, por lo tanto, a la dominación) cuándo sólo dispone para pensarlo y pensarse o, mejor aún, para pensar su relación con él, de instrumentos de conocimiento que comparte con él y que, al no ser más que la forma incorporada de la estructura de la relación de dominación, hacen que ésta se presente como natural" (Bourdieu, 1999. P. 224-225). Esta coerción se presenta como necesaria, pues el habitus ha estructurado la mente de cada individuo y ha logrado

${ }^{1}$ Habitus no puede ser entendido ni traducido, en la literatura sobre Bourdieu, por la palabra española "hábito". No significan lo mismo. Queda hecha la advertencia. 
que su pensamiento, visión, apreciación y acción, es decir todo lo que constituye su práctica, se ajusten a los esquemas sociales con los cuales se involucra a diario. Por lo tanto, la violencia simbólica es una "creencia socialmente inculcada" (Bourdieu 2000) que influye en el sujeto para ver las relaciones de poder como una relación afectiva y no de dominación. En lo que respecta a la relación entre lo femenino y masculino, son las mujeres quienes mantienen esta relación:

"la aplican a cualquier realidad y, en especial, a las relaciones de poder en las que están atrapados, unos esquemas mentales que son el producto de la asimilación de estas relaciones de poder y que se explican en las oposiciones fundamentales del orden simbólico. Se deduce así que sus actos de conocimiento son, por la misma razón, unos actos de reconocimiento práctico, de adhesión tóxica, creencias que no tiene que pensarse ni afirmarse como tal, y que "crea" de algún modo la violencia simbólica que ella misma sufre" (Bourdieu 2000, p. 49).

De todo esto, se desprende que las conciencias no están “engañadas" y que la educación se trate de "salir de la caverna", sino que la violencia simbólica funciona gracias a inclinaciones modeladas por la estructura de la dominación que las produce a ellas.

Las relaciones entre habitus y violencia simbólica confluyen en el mercado lingüístico. Básicamente, este formula los criterios de aceptación y naturalización del poder social de los que interactúan (Bourdieu 1982). En esta lucha por el poder, algunos pierden y otros ganan lo que Bourdieu llama capital simbólico (2000). Es pertinente recordar que estas luchas acontecen como parte del habitus, las relaciones de poder son parte del habitus. De ahí la dificultad para pensar en salidas frente a la descripción de la sociedad que Bourdieu presenta. Sostenemos que las expresiones discursivas, que hemos conceptualizado más arriba, permiten comprender cómo el babitus del profesorado en tanto que sujetos participantes de luchas simbólicas en la sociedad trasmiten hacia el alumnado de un modo no consciente las luchas simbólicas. En otras palabras, las estructuras sociales pueden ser trasmitidas sin mayor conciencia desde el habitus del profesorado hacia el alumnado. Las evidencias concretas de esta teoría debe ser materia de investigaciones que desde la Educación o las Ciencias Sociales se pueden realizar.

\section{GÉNERO Y ESTEREOTIPOS DE GÉNERO}

El género es una construcción social, cultural e histórica que determina la identidad y el comportamiento a través de la vivencia de experiencias, ritos y costumbres atribuidas por el grupo social y su medio (Lamas, 1986; Rubin, 1986). Podemos sostener que el género es socialmente impuesto y sus características son arbitrarias. En ese sentido, pertenece a un sistema cultural y pertenece también a un habitus y sus correspondientes luchas simbólicas. En las luchas simbólicas del género, se sustenta y mantiene la subordinación de las mujeres como consecuencia de relaciones de poder que organizan la sexualidad. Existen, por lo tanto, relaciones de género en la sociedad que responden a luchas simbólicas. Las estadísticas han mostrado que la subordinación de las mujeres es real y responde posiblemente a estas relaciones legitimadas en un mercado lingüístico y encarnadas en un habitus. En términos de Teresita De Barbieri, los sistemas de género-sexo son los conjuntos de prácticas, símbolos, representaciones, normas y valores sociales que las sociedades elaboran a partir de la diferencia sexual anatomo-fisiológica y que dan sentido a la satisfacción de los 
impulsos sexuales, a la reproducción de la especie humana y en general al relacionamiento entre las personas (De Barbieri, 1993, p. 149).

Como construcción social, el género es un concepto que dirige ciertas prácticas sociales, en eso se cifra su utilidad. Con este concepto se establece una dominación que las mujeres aceptan, porque la eficacia del orden social consiste en que se reafirma con la práctica. En esta práctica aparecen los estereotipos, pues de este modo aparecen las relaciones interpersonales, pues el estereotipo es “(...) es un elemento que nos facilita la aprehensión de la realidad más lejana y también la más cercana; esto supone un arma de doble filo, ya que en ocasiones depender de estereotipos puede suponer la conformación de imágenes cognitivas erróneas o en otros casos el encasillamiento en comportamientos determinados, con lo que se permiten conductas distintas llegando a situaciones de censura" (p. 151)

Dicho de otro modo, se trata de ideas que permiten la creación de comunidades y la identificación con ellas. López (2005) sostiene que "El estereotipo es subjetivo y dirige las expectativas de un grupo social, determinando sus opiniones. El origen del estereotipo es emocional y tiene su base en una determinada utilización del lenguaje". El estereotipo tiene la capacidad de relacionar las características físicas de un individuo con una serie de características subjetivas que se esperan de él. Debido a ello, es de "sentido común" que se espere de hombres y mujeres determinados comportamientos respecto de cómo actuar y comportarse. Este código de actuar es lo que sostiene y fomenta la diferencia binaria de hombres y mujeres, y que, a partir de la violencia simbólica, así como el babitus, se inscribe en el cuerpo biológico de cada individuo.

\section{EL ÁMBITO Y EL OBJETO DE ESTUDIO}

Entonces ¿por qué creemos que las Ciencias Sociales y la Educación son las disciplinas que pueden reflexionar sobre las formas cómo las prácticas sociales aparecen y se mantienen, y quizás, reflexionar sobre cómo superarlas? La respuesta es el ámbito que pueden compartir: la institución socializadora de la escuela.

En una entrevista realizada por Didier Eribon para Libération, en 1982, Pierre Bourdieu sostuvo que el discurso puede considerarse la síntesis entre un habitus y un mercado, ambos lingüísticos. Como corolario de esta conceptualización, el discurso integra dos elementos que, respecto de él, funcionan como imbricados: la capacidad de expresión y las normas o estructuras que el individuo ha interiorizado como parte de una sociedad. En este sentido, el discurso no es más que un uso particular del conjunto de normas y de interacción que rigen y que reflejan lo que Bourdieu llama el poder social de los actores, ya sean los dominantes o los dominados.

El discurso no es una creación independiente de una persona ni depende de su entera voluntad. Si asumimos la perspectiva de Bourdieu, entonces las acciones, incluso las más íntimas, solo adquieren sentido en referencia al sistema de relaciones que rigen. De este modo, la elección de determinadas palabras, la elección de qué ideas expresar en el discurso involucra un habitus, es decir, una aceptación de que el discurso personal tiene cierto valor y puede ser recibido de una u otra manera en un mercado lingüístico en que participan las personas. No existe el discurso que pertenezca solo al sujeto, sino que las 
expectativas de aceptación y rechazo, interiorizadas en el proceso de socialización, juegan un rol importante en la construcción de aquel.

Las prácticas sociales, por lo tanto, son acciones que expresan el discurso de la persona, pero que al mismo tiempo expresan las estructuras sociales que rigen. Tanto las prácticas sociales consideradas positivas, así como aquellas que se consideren negativas son posibilidades de acciones que emergen de un habitus que, en principio, no es ni bueno ni malo. Ahora bien, sin duda, las reglas de aceptación o rechazo de un discurso muestran que en el encuentro de los discursos habrá algunos que sean más o mejor aceptados, frente a otros que no tendrán este capital simbólico; por lo tanto, Bourdieu expresa que en el mercado lingüístico se lleva a cabo una lucha por el poder simbólico y, claro, aparece una violencia simbólica (Bourdieu, 2000).

De ahí que nos parece que las investigaciones que pretenden dar cuenta de la reproducción y mantenimiento de las prácticas sociales negativas en las escuelas enfoquen mal el objeto de estudio. Incluso, el lenguaje moral de bueno/malo no sería sino un uso particular de las normas y estructuras que rigen nuestra sociedad, y tampoco estamos seguros de que sea el discurso con mayor capital simbólico. La existencia de los estereotipos de género no puede explicarse por la voluntad de algunas personas, sino que debe explicarse como parte de la estructura que permite, de hecho, la socialización de las personas desde niños o niñas. En otras palabras, el discurso de género se adquiere y defiende a través de los múltiples contrastes y experiencias sociales, en un proceso de aprendizaje y socialización en las normas discursivas del grupo en que el sujeto es producido (Alonso, 2000). En términos de Bourdieu: "La homología de oposiciones y la orquestación más o menos perfecta del habitus favorece un reconocimiento práctico de los intereses" (Bourdieu, 1991, p. 98). Dicho de otro modo, los estereotipos de género son un discurso que funciona y estructura la constitución de muchas personas como sujetos sociales. De ahí que será muy difícil extirparla solo por considerarla, desde otro discurso, como moralmente negativo. Incluso, los actos de habla, que se podrían considerar particulares, no se producen como actos calculadores racionalizados, por el contrario, lo que hacen es expresar un habitus que es un habitus lingüistico. Es decir, la suma de "disposiciones adquiridas, esquema de percepción, y de apreciación de la realidad, así como la actuación en ella, que se han aprendido en un contexto social y en una situación determinada" (Bourdieu, 1991, p. 99).

En lo que respecta a las investigaciones educativas, no puede hablarse de discurso particular, sino de expresiones discursivas. Todo acto o toda acción que pretenda organizar un espacio con un orden que remite a las estructuras sociales que rigen es una expresión discursiva. Si esto ocurre con las ideas, lo mismo ocurre con las elecciones que parecen causales como la ropa, los gustos musicales, los lugares frecuentados, etc.; en el colegio, se tiene que focalizar otras expresiones discursivas además del discurso oral. Esta ampliación es la forma de ser conscientes, como sujetos investigadores, de la amplitud y profundidad de arraigo de una práctica social. 


\section{LA RAZÓN PARA AMPLIAR EL OBJETO DE ESTUDIO: ESTRATEGIAS DISCURSIVAS}

La clarificación de que existen luchas simbólicas por el poder en la sociedad obliga a pensar cómo estas "luchas" no son evidentes como fenómenos sociales. La respuesta es que estas luchas constituyen más bien muchos de los fenómenos sociales. Estos últimos ocurren y son evaluados con referencia a un "sistema de legitimaciones". En otras palabras, las estructuras se naturalizan porque seducen la voluntad y controlan el pensamiento $^{2}$.

Las prácticas sociales que expresan estereotipos de género, por lo tanto, tienen un sistema de legitimación sostenido por hombres y mujeres que estructuran su vida según la confrontación, como dos grupos distintos, entre lo masculino y lo femenino (Van Dijk, 2005). Dicho de otro modo, las prácticas se mantienen, porque es conveniente o deseable que así sea para un grupo social con poder simbólico.

Por lo tanto, el abordaje de cómo se reproducen las prácticas sociales, como por ejemplo los estereotipos de género, requiere el análisis de las expresiones discursivas del profesorado como participantes de un sistema de legitimación social. En este punto, por supuesto, falta una investigación-acción que identifique si las actividades del estudiantado reproducen relaciones de género sociales por influencia de las expresiones discursivas del profesorado. El desarrollo de esta literatura se vuelve más urgente, sobre todo porque las investigaciones sobre el discurso docente, en su gran mayoría, han tenido como objeto de estudio las expresiones orales de los mismos. Como corolario de esta necesidad, habrá que señalar la existencia de violencia simbólica en las aulas, mantenida y propiciada por un sistema de legitimación del cual es poseedor el profesorado.

El carácter de "inadvertido" de las correcciones sociales supone que las estructuras mentales son más que discursos lingüísticos, son también normas y valores, criterios de pragmática y criterios juicio de la misma realidad social. Por supuesto, el discurso de cualquier persona, un docente en este caso, es consecuencia de la información y las verdades compartidas, pero estructuralmente, el discurso va acompañado de la necesidad de mantener el orden para pertenecer al grupo (moral, económico, social) al que se pertenezca de facto. En efecto, nunca aprendemos un lenguaje, sin aprender, al mismo tiempo, las condiciones de aceptabilidad de este lenguaje. Sospechamos que es de este modo como el alumnado aprende a aceptar las relaciones de género en la sociedad.

\section{A MODO DE CONCLUSIÓN}

El artículo pretende mostrar que, desde la lectura de Bourdieu sobre la realidad social, los estereotipos de género ( $\mathrm{y}$, por tanto, la violencia de género y la dominación de las mujeres), son parte de las estructuras conformantes de nuestra sociedad. En ese sentido, toda investigación que tenga como objeto el discurso oral del docente no analiza el mercado lingüístico ni las reglas de aceptación y rechazo de este discurso, por lo tanto, puede que no atisbe la verdadera dimensión del problema. La perspectiva que

${ }^{2}$ El discurso del "bienestar", por ejemplo, seduce a muchos, sino a todos en la actualidad: un trabajo remunerado, un tipo de casa, ciertos productos y marcas, etc. Pareciera que es cuestión de "querer" y trabajar para conseguirlo. Se crean así expectativas y esperanzas. 
proponemos de modo teórico para futuras investigaciones que ocupen la intervención en la realidad es que se debe estudiar toda la gama posible de expresiones discursivas del profesorado que se relaciona directamente con el estudiantado: ambientación del aula, materiales elaborados, repartición de roles en el aula, etc. Esto, porque la dominación simbólica no funciona gracias a conciencias engañadas, sino que forma parte de las estructuras que otorgan sentido a las acciones de los agentes. ¿cómo escapamos al habitus? Según Bourdieu, existe lo que él llama la reinversión simbólica, que consiste en transformar las estructuras mismas del orden simbólico dominante. Es aquí en que se requiere, si deseamos cambiar las relaciones de género actuales por otras, ampliar la conciencia sobre cómo participamos de la sociedad y de sus reglas regentes. Esta tarea pertenece a otro tipo de investigación del que presentamos aquí.

\section{REFERENCIAS BIBLIOGRÁFICAS}

Parker, I. (2010). La psicología como ideología. Contra la disciplina. Madrid: Catarata

Bourdieu, P. (1990). Sociología y cultura. México D.F.: Grijalbo

Bourdieu, P. (2000) La dominación masculina. Barcelona: Anagrama

Lamas, M. (1986). La antropología feminista y la categoría "género". Nueva Antropología, 8(30), 173-198

Rubin, G. (1986). El tráfico de mujeres: Notas sobre la "economía política” del sexo. Nueva Antropología, 8(30), 95-145

Barbieri de, T. (1993). Sobre la categoría género. Una introducción teórico-metodológica. Debates en sociología, (18), 145-169

López, E. (2005). Propuesta para una educación no sexista: Coeducación e igualdad de sexos en el área de Educación Física. Educación física y deportes, 81. Recuperado de: http://www.efdeportes.com/efd169/la-coeducacion-eneducacion-fisica.htm

Alonso, L. (2004). Pierre Bourdieu, el lenguaje y la comunicación: del análisis de los mercados lingüísticos a la denuncia de la degradación mediática. En Alonso, L., Criadro, E. y Moreno, J. (eds.). Pierre Bourdieu, las herramientas del sociólogo. Madrid: Fundamento

Alonso, L. (2000). "Pierre Bourdieu in memoriam (1930-2002). Entre la bourdieumanía y la reconstrucción de la sociología europea". Revista Española de Investigaciones Sociologicas, 97, pp. 9-28.

Van Dijk, T. (2005). Política, ideología y discurso. Quórum Académico, 2(2), 15-47

Aymerich, M y Díaz, L (1989). Estrategias del discurso del profesor. Repeticiones y reformulaciones. Cable 3, s/n, 39-44

Bauman, Z. (2007). Los retos de la educación en la modernidad líquida. Barcelona: Gedisa.

Berger, P. y Luckmann, T. (1995). La construcción social de la realidad. Buenos Aires: Amorrortu

Bernstein, B. (2001). La estructura del discurso pedagógico. Madrid: Morata 
Bolaños, L. y Jiménez-Cortés, R. (2007). La formación del profesorado en género. Revista de investigación educativa, 25(1), 77-95

De Longhi, A. (2000). El discurso del profesor y del alumno: análisis didáctico en clases de ciencias. Enseñanza de las ciencias, s/n, 201-216

Edwards, V. (1989). Conocimiento escolar e identidad del maestro: dos aproximaciones etnográficas. Documento de trabajo $\mathrm{N}^{\circ}$ 6. Santiago: PIIE

Flores, R. (2017). Discursos y prácticas sobre masculinidad y feminidad en la escuela: El caso del Colegio Preuniversitario Trilce Los Olivos, 2015-2016. [Tesis de grado]. Tomado de http://cybertesis.unmsm.edu.pe/handle/cybertesis/7352

Guevara, Y. (2014). Percepción de violencia de género en los alumnos de la Universidad Nacional de Cajamarca sede Chota. [Tesis de grado]. Recuperado de http:/ / repositorio.unc.edu.pe/handle/UNC/175

Hernández, R., Fernández, C. \& Baptista, L. (2003). Metodología de la Investigación. México, D.F.: McGraw-Hill.

INEI. (2017). Violencia contra las mujeres, niñas y niños. Encuesta demográfica y de salud familiar-ENDES.

Martínez-Otero, V. (2007). Modelo pedagógico del discurso educativo y su proyección en la calidad docente, discente e institucional. Revista Iberoamericana de educación, 43(2), 30-35.

Peralta, B. (2015). Relaciones interpersonales de género en los estudiantes de secundaria de la i.e.p. "José Gabriel Condorcanqui" del distrito de Carmen Alto 2014-2015. Tesis de Licenciatura. Universidad Nacional de San Cristóbal de Huamanga. Ayachuco, Perú. Tomado de http://repositorio.unsch.edu.pe/handle/UNSCH/965

Rosales, F. (1998). Análisis del discurso pedagógico y natural. Hacia un modelo de interpretación del discurso del aula. Centro Virtual Cervantes. Asele, actas IX.

Schutz, A. (1792). Fenomenología del mundo social. Buenos Aires: Paidós.

Zimmermann, L. (1997). La comunicación en el aula. El metacurrículum. Facultad de ciencias políticas y sociales UNcuyo. Mendoza.

\section{CITAR COMO:}

Vargas Huanca, G., \& Quispe Pérez, Ítalo J. (2019). Discurso y expresiones discursivas del profesorado ¿cómo se reproducen los estereotipos de género? Puriq, 1(01), 48-56. https://doi.org/10.37073/puriq.1.01.11 